\title{
RESÚMENES EN ESPAÑOL
}

\section{ChILE’s ECONOMIC Growth}

\author{
KLaus Schmidt-HebBel \\ Central Bank of Chile
}

El elevado crecimiento del PIB per cápita de Chile observado entre 1991 y 2005 , de 4,1\% promedio anual, excedió significativamente el crecimiento promedio mundial y constituyó también un claro quiebre con el crecimiento pasado del país. ¿Cuánto del crecimiento reciente constituye crecimiento de tendencia y cuánto es cíclico, influido por las condiciones externas que enfrenta el país? ¿Cuáles son los principales determinantes del crecimiento de largo plazo y de las fluctuaciones cíclicas de acuerdo con la literatura empírica reciente? ¿Cuáles son las principales fortalezas y debilidades para el crecimiento de Chile y, basado en las anteriores, cuáles son las reformas propuestas por los estudios técnicos y los programas presidenciales más recientes? Esta revisión responde a las preguntas anteriores, comenzando con los hechos que caracterizan el crecimiento promedio y la volatilidad del crecimiento, a nivel agregado, regional y sectorial. Luego se revisan los estudios del crecimiento de tendencia en Chile, que incluyen desde las descomposiciones de las fuentes del crecimiento hasta los estudios econométricos $y$ de simulaciones de los determinantes profundos del crecimiento, basados en evidencia internacional de datos de paneles y series de tiempo nacionales, así como en investigación focalizada en reformas individuales. A continuación se presentan estudios sobre las fluctuaciones de corto plazo y las recuperaciones cíclicas, también basados en evidencia internacional y nacional. Este número de Cuadernos de Economía publica seis nuevos y relevantes trabajos de investigación sobre el crecimiento de Chile. Luego el artículo revisa selectivamente las propuestas de políticas y reformas en apoyo de un mayor crecimiento, presentadas por autores académicos, organizaciones internacionales y candidaturas presidenciales en el año 2005. Un epílogo con breves reflexiones sobre el crecimiento potencial de Chile y los principales retos de políticas en sustento de un mayor crecimiento cierra este artículo. 


\title{
COMPLEMENTARITIES BETWEEN INSTITUTIONS AND \\ Openness in EConomic Development: Evidence FOR A PANel OF COUNTRIES
}

\author{
CÉSAR CALDERón \\ The World Bank \\ RODRIGO FUENTES \\ Central Bank of Chile
}

El objetivo del presente trabajo es evaluar las fuentes de crecimiento de Chile y de las economías mundiales en los últimos años, poniendo énfasis en el rol de la complementariedad de las políticas económicas. Por tal motivo, evaluamos los determinantes del crecimiento económico para una muestra de 78 países y con información para el período 1970-2000. A diferencia del enfoque de Gallego y Loayza (2002), el presente trabajo contrasta directamente la interacción existente entre las políticas de liberalización comercial y financiera con: a) las condiciones iniciales de la economía; b) con las políticas de capital humano, y c) con la calidad de la institucionalidad. 


\title{
Regional Convergence in Chile: New Tests, Old RESUlts
}

\author{
ROBERTO DUNCAN \\ University of Wisconsin \\ RODRIGO FUENTES \\ Banco Central de Chile
}

Los tests tradicionales de convergencia evalúan implícitamente la hipótesis de raíz unitaria en las series de producto per cápita. A pesar de que bajo la hipótesis nula estos estadísticos no tienen distribuciones asintóticas ni valores críticos estándares, la gran mayoría de los trabajos sobre el tema los emplean con los consiguientes problemas de inferencia. El objetivo del trabajo es determinar la existencia de convergencia en niveles en los PIB e ingresos de las regiones de Chile, empleando no sólo la batería tradicional de tests (corte transversal y paneles de datos), sino fundamentalmente los más recientes test de raíz unitaria para paneles y así poder hacer inferencia apropiadamente. Se analiza, además, la convergencia en dispersión construyendo intervalos de confianza para la varianza de la producción e ingresos regionales y evaluando la presencia de asimetrías o formaciones de "clubes" regionales mediante un test no paramétrico de multimodalidad. Se concluye que: (1) la evidencia respalda la hipótesis de convergencia $\beta$ absoluta, tanto en PIB como en ingresos; (2) las tasas de convergencia de ingresos son mayores a las del PIB, pero ambas son relativamente bajas comparadas con la evidencia internacional; (3) no obstante, la tasa de convergencia condicional se ve estadísticamente incrementada si se controla por participaciones sectoriales como minería; (4) a pesar de que las distribuciones empíricas del PIB e ingresos muestran aparentes “clubes” regionales, estos hallazgos no son estadísticamente significativos; (5) se encuentra evidencia a favor de convergencia 6 en PIB para el período 1960-2000, mientras que la dispersión de ingresos (estadísticamente) no ha cambiado durante el período 1987-2000. 


\title{
Sources of Growth AND BeHAVIOR OF TFP IN CHILE
}

\author{
RODRIGO FUENTES \\ MaURicio LaRraín \\ Klaus SchmidT-HeBbel \\ Central Bank of Chile
}

\begin{abstract}
Este trabajo presenta nuevas estimaciones de las fuentes del crecimiento de Chile y nuevas medidas de factores de producción y PTF durante el período 19602005. Usando una función de producción Cobb-Douglas, el trabajo presenta ocho series de PTF basadas en combinaciones alternativas de medidas de factores productivos. El trabajo también reporta evidencia econométrica acerca del comportamiento de la PTF durante 1960-2005. La dinámica de la PTF es afectada por variables cíclicas (términos de intercambio y subvaluación del tipo de cambio real), variables relacionadas con políticas estrcturales (estabilización macroeconómica y progreso en reformas microeconómicas) y también por efectos positivos de interacción entre desempeño macro y políticas micro.
\end{abstract}

\section{Productividad Sectorial en Chile: 1986-2001}

\author{
RODRIGO VERGARA \\ Universidad Católica de Chile \\ ROSARIO RIVERO \\ Universidad Católica de Chile
}

El objetivo de este artículo es hacer estimaciones de productividad total de los factores a nivel sectorial. El principal desafio que se enfrenta es la ausencia de series de stock de capital por sectores para un período largo de tiempo. Sólo se tienen datos de stock de capital para el período 1996-2001. Se diseña una metodología para, a partir de dichos datos y otra información, generar cifras de stock de capital para el período 1986-1995.

Con ello, más un ajuste por utilización de las series de capital y un ajuste de calidad relativa para las series de trabajo, se hacen estimaciones de PTF sectorial para 1986-2001. Según estos cálculos, el mayor incremento de productividad en este período se ha producido en el sector comercio. Esto coincide con estudios para otros países, que muestran que los sectores con mayor incremento de la productividad corresponden a aquellos que usan intensivamente la tecnología de la información. 


\title{
Micro EfFiciency AND AgGregate Growth IN ChILE
}

\author{
RAPHAEL BERGOEING \\ Universidad de Chile \\ ANDREA REPETTO \\ Universidad de Chile
}

\begin{abstract}
En este trabajo analizamos las fuentes microeconómicas de la eficiencia agregada, principal determinante del crecimiento agregado. Con este fin, estimamos y caracterizamos la productividad total de factores utilizando datos chilenos de plantas manufactureras durante el período 1980-2001. Al descomponer los cambios en eficiencia agregada que resultan de reasignación productiva y de variaciones al interior de las plantas, encontramos que la primera explica casi la totalidad de la mayor productividad alcanzada durante las últimas dos décadas. Estas ganancias por reasignación, a su vez, se generan principalmente por la entrada de plantas nuevas más eficientes.
\end{abstract}

\section{Procyclical Productivity in Manufacturing}

\author{
LUCAS NAVARRO \\ University of London \\ RaIMUNDO Soto \\ Pontificia Universidad Católica de Chile
}

\begin{abstract}
Estudiamos el comportamiento cíclico de la productividad en ochenta industrias del sector manufacturero chileno entre 1979 y 2001. Encontramos que a nivel de sectores la productividad es inambiguamente procíclica, pero que en el agregado es acíclica. Damos una explicación analítica y empírica para este fenómeno. Finalmente usamos un modelo econométrico que permite cuantificar la contribución al crecimiento de productividad de cuatro componentes. Los resultados indican que la mitad del crecimiento de productividad se debe a cambios tecnológicos, lo que apoya la hipótesis que los shocks de oferta son la principal fuente del ciclo económico en Chile. El resto del crecimiento de productividad se explica por la reasignación de recursos desde sectores con baja productividad hacia aquellos más productivos y por el aprovechamiento de economías de escala. Las variaciones en el uso de insumos resultan poco significativas.
\end{abstract}

